la revue La revue pour l'histoire du CNRS

POURLHISTOIRE DU CNRS $24 \mid 2009$

Soixante-dixième anniversaire du CNRS

\title{
Nom de code : FSD
}

Par sa richesse scientifique et intellectuelle et son potentiel technique et humain, le CNRS suscite bien des convoitises.

Joseph Illand

\section{(2) OpenEdition}

Journals

Édition électronique

URL : https://journals.openedition.org/histoire-cnrs/9142

DOI : 10.4000/histoire-cnrs.9142

ISSN : 1955-2408

Éditeur

CNRS Éditions

Édition imprimée

Date de publication : 5 octobre 2009

ISSN : 1298-9800

Référence électronique

Joseph Illand, « Nom de code : FSD », La revue pour l'histoire du CNRS [En ligne], 24 | 2009, mis en ligne le 05 octobre 2009, consulté le 20 mai 2021. URL : http://journals.openedition.org/histoire-cnrs/9142 DOI : https://doi.org/10.4000/histoire-cnrs.9142

Ce document a été généré automatiquement le 20 mai 2021.

Comité pour l'histoire du CNRS 


\section{Nom de code : FSD}

Par sa richesse scientifique et intellectuelle et son potentiel technique et humain, le CNRS suscite bien des convoitises.

\section{Joseph Illand}

Du concept de « guerre froide » à celui d'intelligence économique

1 Jusqu'à la chute du rideau de fer en 1989, la fonction de sécurité de défense s'est déclinée sur fond d'espionnage et de guerre froide. Objectif : préserver les domaines de recherche à applications civiles et/ou militaires, sécuriser les transferts de technologie vers des pays étrangers, et empêcher les risques de détournement d'armes de destruction massive et de leurs vecteurs (nucléaire, biologique ou chimique). Si la vigilance est longtemps restée tournée vers l'Est, l'attention s'est sensiblement accentuée vers les «États voyous» et les risques de prolifération d'armes de destruction massive et plus récemment vers le terrorisme (11 septembre 2001 oblige). En parallèle, les risques de simple «pillage technologique » ont fait l'objet d'une prise de conscience accrue, dans un contexte d'exacerbation de la concurrence scientifique, technologique et économique internationale. Le concept de "défense économique active ", volet défensif de l'intelligence économique, s'est ajouté au vocabulaire.

Le patrimoine scientifique sous haute protection

2 Le nouveau code pénal ${ }^{1}$ est venu en 1992 "sanctuariser » le patrimoine scientifique et économique en le mettant au rang des «intérêts fondamentaux de la nation " (article 410.1). Pour le CNRS, établissement public de recherche, le continuum existe entre "son patrimoine scientifique» et celui de la nation. Par patrimoine scientifique et technique, il faut donc comprendre à la fois les intérêts nationaux dans leurs dimensions commerciale, industrielle, scientifique, militaire... mais aussi les intérêts de l'unité ou de l'établissement : renom et image de la structure, qualité et renom des chercheurs et autres personnels, qualité des recherches et de l'enseignement, crédibilité scientifique des résultats, conformité des travaux à l'éthique, portefeuille de brevets, potentiel technique... Au niveau national, il est naturel que les investissements financiers et humains pour la recherche le soient au bénéfice du pays et non de puissances ou sociétés étrangères. Il en va en particulier de la préservation de nos acquis et avances technologiques, de l'économie du pays et de son rayonnement et aussi de l'emploi, 
actuel et futur. Au titre des atteintes au patrimoine on rangera ainsi les transferts indus de technologie ou de savoir-faire au profit de sociétés ou pays étrangers, mais aussi les détournements de propriété intellectuelle, lorsque l'employeur se trouve privé de ses droits de propriété intellectuelle par indélicatesse du chercheur, permanent ou stagiaire. Ces atteintes peuvent se produire, parfois avec l'implication consciente du chercheur (sur des motivations d'enrichissement personnel ou simplement de "reconnaissance»), le plus souvent avec sa contribution inconsciente (maladresse, insouciance...), et bien sûr aussi par espionnage délibéré (récupération de données, de savoir-faire...) soit physiquement (via des stagiaires ou par intrusion physique et vol), soit plus subtilement par compromission de données informatiques (stockées ou échangées). On n'insistera jamais assez sur la nécessité de protéger ses données sensibles, sur son poste de travail, sur son portable, sur ses clés USB, ou lors de transmissions par messagerie.

De la sécurité informatique à la sécurité des systèmes d'information. À l'écoute et sur écoute...

3 La problématique de la sécurité informatique est déjà présente dans les préoccupations nationales dès les années 1970 (création du Service central de la sécurité des systèmes d'information au sein du SGDN en 1976, publication de la loi Informatique et Liberté en 1978...). Les nouvelles technologies de l'information et de la communication induisent des risques touchant à la confidentialité des données (atteintes possibles au patrimoine scientifique mais aussi à la sphère privée de l'individu), à l'intégrité des données et à leur disponibilité (avec les impacts possibles sur les capacités des systèmes et leur légitimité). Sous l'impulsion de Philippe Schreiber, alors FSD du CNRS, la fonction «FSD » intègre cette problématique, avec la création en 1995 d'un chargé de mission SSI au CNRS et son rattachement au FSD. La même année est lancée par le FSD, le bulletin "Sécurité informatique " dont l'audience dépasse maintenant largement le CNRS. Une nouvelle dynamique sera encouragée en 2006 avec la rédaction et la publication en fin d'année de la Politique de sécurité des systèmes d'information (PSSI) du CNRS, la refonte de la charte utilisateur (janvier 2007), la montée en puissance au niveau local du réseau des responsables de la SSI (coordinateurs régionaux et chargés de la SSI dans les laboratoires).

De la protection des biens matériels et immatériels à la protection des individus

4 L'accroissement des risques terroristes voire sanitaires a conduit le CNRS à mettre en place un dispositif de vigilance et de conseil à l'attention des missionnaires partant dans des pays à hauts risques pour les visiteurs. Cette procédure, menée en étroite liaison avec les ambassades et la cellule de veille et de crise du ministère des Affaires étrangères, peut aller jusqu'à l'annulation de missions, voire le rapatriement de missionnaires. Le service du FSD intervient aussi de plus en plus dans des cas de mise en cause grave de personnels du CNRS (menaces de mort, chantage, harcèlement, atteintes à l'honorabilité) et ce, dans un contexte où certaines recherches peuvent faire l'objet de controverses exacerbées au sein de la société civile nationale.

Le FSD, prophète ou ange gardien de la science?

5 L'ordonnance de 1959 avait institué la mise en place de Hauts fonctionnaires de défense dans les ministères dont celui chargé de la recherche. Leur ont été adjoints des Fonctionnaires de sécurité de défense dans les départements ministériels et les établissements publics sous tutelle. Le CNRS dispose depuis les années 1970 d'un FSD2 Assisté de quelques collaborateurs, dont un expert de la sécurité des systèmes 
d'information, ce FSD s'appuie sur le terrain sur les délégations régionales du CNRS mais aussi sur les troupes locales que constituent les responsables de la SSI en laboratoires. Au-delà de son rôle quotidien de contrôle de procédures liées aux échanges internationaux (accueil de stagiaires et recrutements de ressortissants étrangers, coopérations internationales, missions en pays à risques...), le FSD réalise un travail de veille, de détection et d'évaluation des risques. En prime, il conseille, informe, sensibilise et prêche, entre naïveté et paranoïa, la prise de conscience et l'esprit de vigilance auprès des populations du CNRS. Vaste challenge !

«Baisers amers de Russie»

1981: un espion russe, Vladimir Vetrov, se retourne contre l'URSS et livre à la DST, sous son nom de code Farewell, des informations de première importance. L'Académie des sciences de l'URSS, liée aux services de renseignement, était notamment chargée d'envoyer des stagiaires à l'étranger pour des opérations d'intelligence économique. L'espionnage russe dans les domaines les plus sensibles de la recherche et de la technologie, notamment par le biais d'envoi de stagiaires étrangers dans les laboratoires connaît alors son apogée. Ces révélations vont conduire à l'expulsion en 1983 de 47 diplomates soviétiques. Elles vont surtout déclencher une véritable prise de conscience des vulnérabilités de la recherche civile, beaucoup moins protégée que la recherche ou l'industrie militaire, et de la fragilité des laboratoires.

Les intérêts fondamentaux de la nation

«Les intérêts fondamentaux de la nation s'entendent au sens du présent titre de son indépendance, de l'intégrité de son territoire, de la forme républicaine de ses institutions, des moyens de défense et de sa diplomatie, de la sauvegarde de sa population en France et à l'étranger, de l'équilibre de son milieu naturel et de son environnement et des éléments essentiels de son potentiel scientifique et économique et de son patrimoine culturel.» Code pénal - article 410-1 - Des atteintes aux intérêts fondamentaux de la nation (Livre IV - titre I)

\section{NOTES}

1. Loi $92-686$ du 22 juillet 1992 intégrant le livre IV du Code pénal qui introduit le concept nouveau d'« atteintes aux intérêts fondamentaux de la nation » (article 410-1). 2. Daniel Pouget (1977-1980), Marc Girardon (1980-1993), Philippe Schreiber (1993-2000), Alfred Schwenck (2000-2003), et Joseph Illand (depuis 2003). Traditionnellement depuis 1980 et jusqu'à présent, ces FSD ont été jusqu'à présent des officiers généraux (Armée de l'air, puis Armement pour l'actuel titulaire). 


\section{RÉSUMÉS}

Divulgation de secrets, fraudes, pillage technologique et informatique, atteinte à l'intégrité des agents, menaces sur la sécurité interne... Depuis plus de trente ans, le fonctionnaire de sécurité de défense (FSD) du CNRS veille, sur veille et protège...

\section{AUTEUR}

JOSEPH ILLAND

Joseph Illand est fonctionnaire de sécurité de défense au CNRS depuis 2003. 